\title{
Global Approach for Hybrid Vehicle Optimal Control
}

\author{
Julien Scordia ${ }^{1}$, Rochdi Trigui ${ }^{2}$, Matthieu Desbois-Renaudin ${ }^{3}$, \\ Bruno Jeanneret ${ }^{4}$, and François Badin ${ }^{5}$ \\ ${ }^{1}$ LMS Imagine, julien.scordia@free.fr \\ ${ }^{2}$ French National Institute for Transport and Safety Research, rochdi.trigui@inrets.fr \\ ${ }^{3}$ CEA Grenoble France, matthieu.desbois-renaudin@cea.fr \\ ${ }^{4}$ French National Institute for Transport and Safety Research, bruno.jeanneret@inrets.fr \\ ${ }^{5}$ IFP France, francois.badin@ifp.fr
}

\begin{abstract}
In this paper, we describe the global approach that has been developed at INRETS-LTE for hybrid powertrain sizing, consumption gain evaluation, and optimal control. The procedure is described in the particular case of a two clutch parallel hybrid architecture, but could apply to any other configuration. Firstly the context and the common issues related to this subject are described. Then our global approach is detailed: sizing procedure, offline global optimization process, method for obtaining suboptimal online energy management laws, and the use of these laws on the test bench. Simulation or optimization results are presented at each step of the procedure.
\end{abstract}

\section{Keywords}

hybrid vehicle, powertrain sizing, consumption minimization, two clutch parallel hybrid

\section{INTRODUCTION}

The green house effect has been discussed for a long time and is now recognized as being an important problem by the majority of scientists and politicians. Consequently, countries have been committed themselves to reduce $\mathrm{CO}_{2}$ emission, particularly in the ground transportation field, because its share is rather important (20 to $30 \%$ depending on countries). One secondary reason to this reduction is the increase of fuel cost, which is directly related to $\mathrm{CO}_{2}$ emissions. Hybridization of terrestrial vehicles is one of short to mid-term solutions being proposed. A wide range of hybrid solutions can be implemented, from the simple start/stop vehicle (e.g. Citroën C3) to the full and complex serial/parallel configurations (e.g. Toyota Prius).

The fuel consumption potential gain of these different architectures has to be carefully evaluated, taking into account the vehicle usage. To be able to define the best solutions in terms of fuel economy for a given dynamic performance specification book, both simulation and optimization processes have to be performed. It is the association between these two approaches that is dealt in the first part of this paper. In the second part, the results obtained on the dynamic test bench of LTE are presented.

The purpose of this paper is to depict the globality of our procedure. To obtain more details about particular points, the reader should consult the other publications of the same authors: [Scordia et al., 2004; Trigui et al., 2004, 2007].

\section{QUALITATIVE DESCRIPTION OF THE PROBLEM}

The principle of hybrid vehicles is to associate two energy sources on board. Strictly speaking, a conventional thermal vehicle is a hybrid one. But our choice is to speak of hybrid vehicles when at least one of the following functions is implemented:

(1) Start/stop: the ability to shut down the internal combustion engine (ICE) when the vehicle is stopped;

(2) Brakes regeneration: the vehicle kinetic or potential energy recovery due to reversible power electronics;

(3) "Boost": the possibility to assist the ICE with the electric motor (EM) during the takeoff.

(4) Electric mode: the ability to drive the vehicle only with the EM.

(5) Auxiliaries' energy management optimization: the choice of the battery charge instants and the amount of this charge: it implies to allow a deeper battery discharge than usually tolerated in the conventional vehicles (from $13.8 \mathrm{~V}$ to $14.2 \mathrm{~V}$ for the open circuit voltage).

The influence of these possibilities on the fuel consumption is examined in $\S 5$.

In the scope of this paper, we focus on the two clutch parallel hybrid architecture (Figure 1), which permits all the possibilities previously described. Its main ad- 


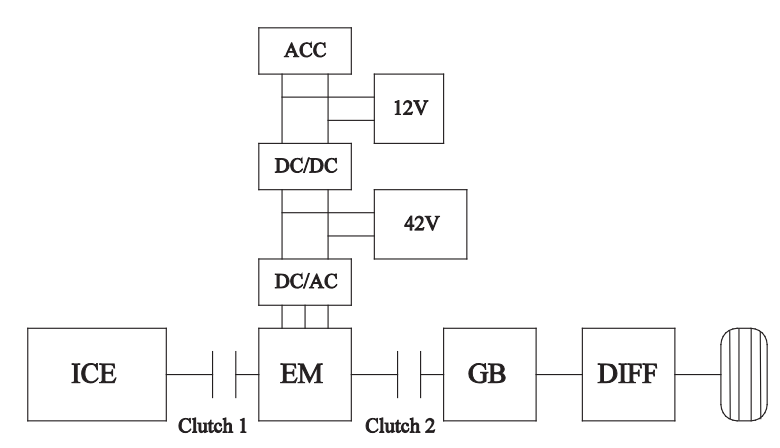

Fig. 1 Two clutch parallel Hybrid vehicle in a 12/42 $\mathrm{V}$ electrical architecture

vantages are:

(1) Inertial ICE start, that is to say the ability to start the ICE with the vehicle kinetic energy, beforehand taken off by the EM;

(2) Better brakes regeneration potential, due to the suppression of the ICE drag torque.

But its drawbacks are:

(1) Complex coordination between the different elements: clutches, EM, ICE;

(2) the breaking of the torque transmission during gear shifting;

(3) belt accessibility.

The goal of this work is to minimize the fuel consumption of the hybrid drivetrain, while satisfying the requirements of dynamic performance. To obtain the greatest possibilities of control, we consider robotized gearbox and clutches. All these components must be controlled by the embedded intelligence, the driver must not interact. It corresponds to the following functions:

(1) Torque repartition between the ICE and the EM;

(2) Clutch C1 and C2 opening/closing;

(3) Gearbox ratio switching.

The behaviour of these components and their interaction acts on fuel consumption. Our purpose is to control them optimally so as to minimize the criterion of the problem that is fuel consumption.

It is a common practice to evaluate vehicle consumptions on speed versus time driving schedules. For example, the normalized New European Driving Schedule (NEDC) is represented on Figure 2 [EEC, 1990]. The NEDC cycle is not representative of mean driving conditions, because of very steady speeds, and also because the compelled gear shift strategy is defined by the standard [EEC, 1990] and entails low ICE rotation speeds. It will be used in the following paragraphs. More realistic driving schedules have been elaborated at INRETS, for example HYZEM and ARTEMIS ones [Andre, 1997, 2002], which are used in $\S 3$. They result from two measurement campaigns lead across Europe on light duty vehicles. Each campaign has led

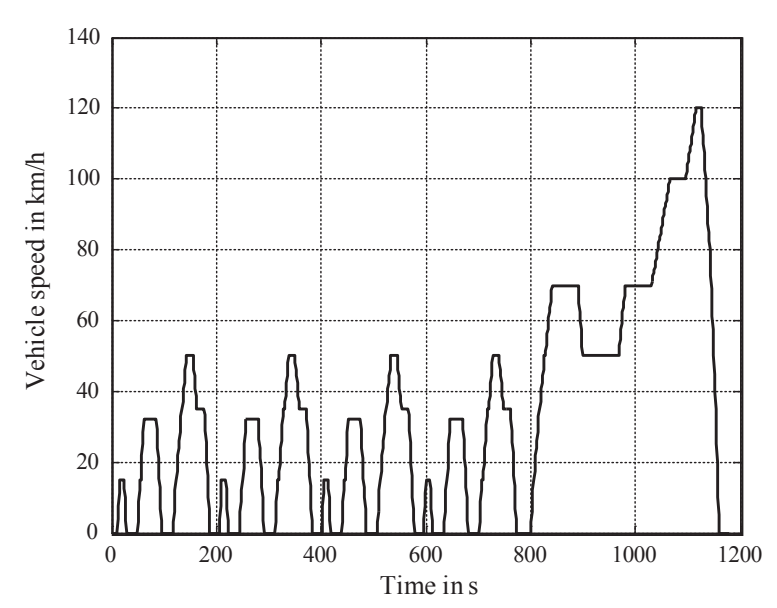

Fig. 2 New European driving cycle

to several driving schedules representing a given vehicle usage: generally urban, road, and highway.

As the considered hybrid vehicle can work in all-electric mode, the first idea to minimize consumption is to never start the ICE, unless it is compulsory to obtain the desired dynamic performances.

But then the battery of the vehicle would be quickly fully discharged. If the vehicle does not offer gridcharging possibility, that is to say if it is a "chargesustaining" hybride vehicle (by opposition to "chargedepleting"), it is not admissible. Then we must control the battery State Of Charge (SOC) so as to keep it in a range of few percents of the battery total capacity.

If the future rolling conditions of the vehicle are known in advance (that is the case if we evaluate the fuel consumption on a given driving schedule), we obtain a significant advantage for the determination of the optimal control of the drivetrain. As the amount of knowledge about the problem is larger, our possibility to minimize the consumption is greater. So it is particularly suited for optimization purpose. This approach with known-in-advance driving schedule is called offline global optimization approach. At the opposite, the online approach does not suppose prerequisite knowledge of the future: it is the case in practice, so it was the final goal of our work $\S 4$.

Similarly to the distinction online/offline, it is a common practice to differentiate the backward and the forward modelling approach:

(1) In the backward approach, the target speed and corresponding torque are strictly imposed to the wheels. There is no speed control. This formalism is adapted to a global optimization tool that we have developed, called KOALA [Scordia et al., 2004], because it is difficult, if not impossible, to perform exact optimization (not heuristic) with dynamic speed control. One could note that this approach is used in the energy oriented software 
Advisor created at NREL [Advisor, 2000].

(2) A forward approach, which is causal and often more accurate since it takes into account transient phenomena and can be easily converted into a real time process. A tool has been developed by LTE, in Matlab/Simulink language, called VEHLIB [Trigui et al., 2004]. It is well-suited for vehicle dynamic performances evaluation.

\section{GENERAL DESCRIPTION OF THE GLOBAL APPROACH}

We present here a method, which can be applied to any type of vehicle, based on a combined concept which uses the two separate tools, KOALA and VEHLIB. This work is split in four steps, with the following goals:

(1) First step $(\S 4)$

Sizing of the components of a hybrid vehicle, with respect to a given specification book. The dynamic performance criterion is the $0-100 \mathrm{~km} / \mathrm{h}$ acceleration time. We also defined a minimum ICE power to be able to continuously drive the vehicle at $110 \mathrm{~km} / \mathrm{h}$ on a $5 \%$ slope, which is a common constraint when sizing a hybrid vehicle [Paganelli, 1999; Akihiro et al, 1999]. The sizing can be done with or without downsizing of the ICE. The computations are performed using VEHLIB.

(2) Second step $\left({ }^{\circ} \S 5\right)$

Evaluation of the consumption potential gain of the previously sized hybrid vehicle, with respect to an a priori known normalized or real use driving schedule. "Potential" means that the computed fuel consumptions are offline optima. They cannot be reached online, the main reason is that online the driving schedule is not known in advance. This stage is carried out with our backward/offline KOALA optimization software.

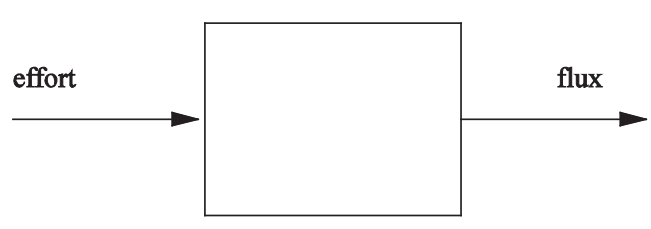

Flux-imposing passive element

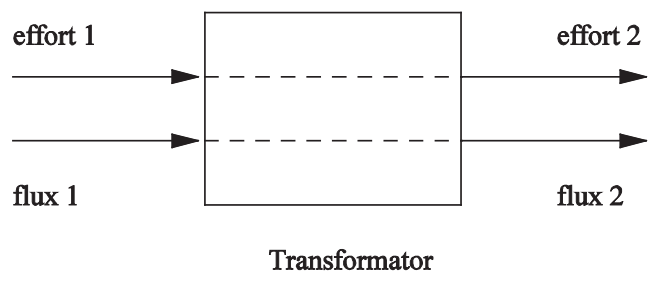

(3) Third step (§ 6)

Elaboration of online energy management laws, using the instantaneous optimization results given by KOALA. These results make up a learning base to construct a radial-basis-functions (RBF) neural network, that is then used in the forward/ online simulation tool VEHLIB.

(4) Fourth step $(\S 7)$

A version of VEHLIB has been dedicated to the dynamic test bench of INRETS-LTE, to perform a Hardware-In-the-Loop (HIL) simulation. The fuel consumption measured on this bench is the final goal of our work.

The consumption potential gain of second step decreased when adapting the optimization results to an online approach. So there is a consumption increase between steps 2 and 3 . This is also the case between steps 3 and 4, because of the differences between the online modelling tool VEHLIB, and the real world (dynamic test bench).

\section{FORWARD SIMULATION TOOL VEHLIB 4.1 Simulation formalism}

The primary goal of VEHLIB is to develop an effective tool for predicting the dynamic performance and energy consumption of any type of vehicle. This objective leads us to the block diagram formalism, with inputs and outputs that essentially represent the effort and flux variables (Figure 3), as it is the case in bond graph formalism [Dauphin Tanguy, 1999]. For a given subsystem of the vehicle, the adopted formalism allows several levels of modelling, of different levels of complexity. In the frame of this work, VEHLIB has been used to size some hybrid vehicles.

\subsection{Sizing procedure}

The objective is to replace the conventional power-

Fig. 3 Example of elements in block diagram formalism

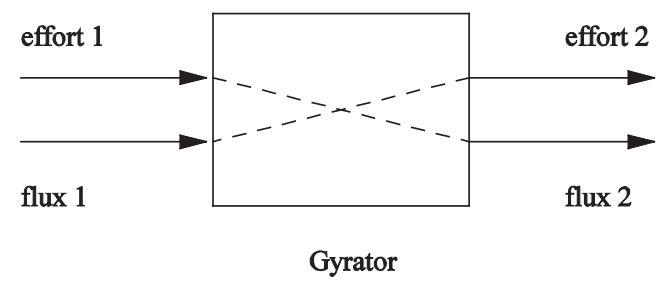


train of a classical thermal vehicle with a hybrid powertrain. This replacement procedure is called hybridization; it consists in:

(1) Keeping the same vehicle chassis and body;

(2) Adding an electric motor of a chosen power;

(3) Adding a battery with a power adapted to the previously chosen electric motor. If the battery has not the requisite battery voltage to use the electric motor, we add a convenient DC/DC boost converter;

(4) Adapting or not (see $\S 4 \mathrm{C}$ ) the internal combustion engine so as to meet the specification book requirements.

For the rest of this article, the original thermal vehicle is called "reference vehicle".

\subsection{Specification book}

Once the electric motor maximum power has been chosen, there are two possibilities when performing step 4 of the hybridization of the reference vehicle:

The first one, keep the same internal combustion engine than the reference vehicle one. Generally this results in an improvement of the vehicle dynamic performances in comparison with the reference vehicle ones. The second one, adapt the internal combustion engine power, so as to reach the reference vehicle dynamic performances, and keep reasonable steady-state performances on highway. This generally results in a downsizing of the engine.

For the two possibilities, the battery is supposed to be charged enough to maintain its nominal power capabilities. The decrease in dynamic performances due to a possibly discharged battery is evaluated but not taken into account during the sizing procedure.

For the rest of the document, the reference vehicle is a Renault Clio $1.51 \mathrm{dCi}$, which characteristics are given in Table 1.

Table 1 Renault Clio 1.5 DCI characteristics

\begin{tabular}{ll}
\hline Mass without powertrain $(\mathrm{kg})$ & 780 \\
\hline $\begin{array}{l}\text { Internal Combustion Engine } \\
\text { type }\end{array}$ & $\begin{array}{l}\text { Compression ignition } \\
\text { Common rail direct injection }\end{array}$ \\
\hline $\begin{array}{l}\text { Rolling/aerodynamic } \\
\text { ressitance }(\mathrm{N})(\mathrm{v} \text { in } \mathrm{m} / \mathrm{s})\end{array}$ & $85+1.38 . \mathrm{v}+0.402 . \mathrm{v} 2$ \\
\hline Wheel radius $(\mathrm{m})$ & 0.296 \\
\hline Gearbox ratios & I: $3.46 ;$ II: $1.87 ; \mathrm{III}: 1.15 ;$ \\
\hline Differential gear ratio & 3.68 \\
\hline
\end{tabular}

The dynamic performances of the sized vehicles are shown on Figure 4 and Figure 5 . The $0-100 \mathrm{~km} / \mathrm{h}$ dynamic performance given by the manufacturer (Renault) are reported on the ordinate axis, for the refer- ence vehicle, the least, and the most powerful existing Clio. Two curves are computed thanks to VEHLIB: performances with charged or fully discharged battery. This last case corresponds to the worst possible situation, and might never happen. Note that there is a 1.5 second gap between the official performance given by Renault (yellow point) and the VEHLIB simulation. This may be due to the fact that the measured engine maximum power is $10 \%$ lower than the Renault published results ( $54 \mathrm{~kW}$ on the engine test bench of LTE against $60 \mathrm{~kW}$ announced by Renault).

Without downsizing (Figure 5), the performances remain acceptable, whatever the battery state of charge may be. Whereas with downsizing (Figure 4), when the battery is discharged, performances decrease highly and could be largely worse than the least powerful vehicle version.

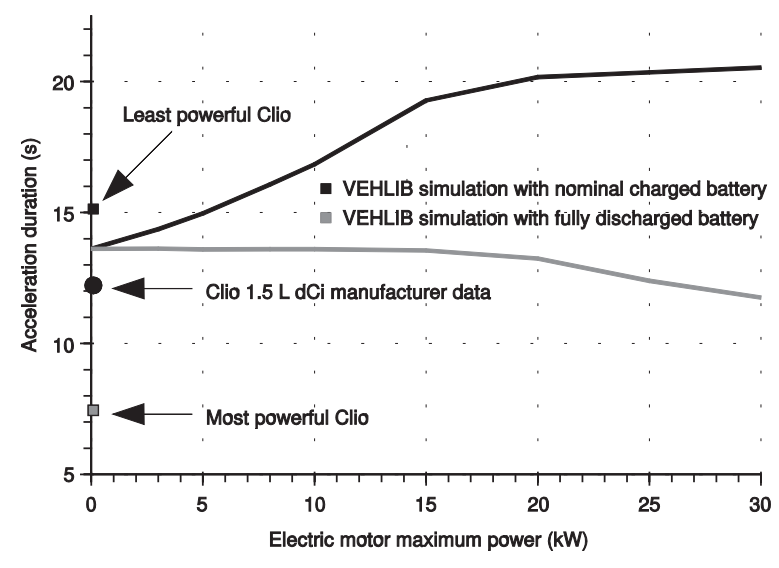

Fig. 4 0-100 km/h acceleration with ICE downsizing

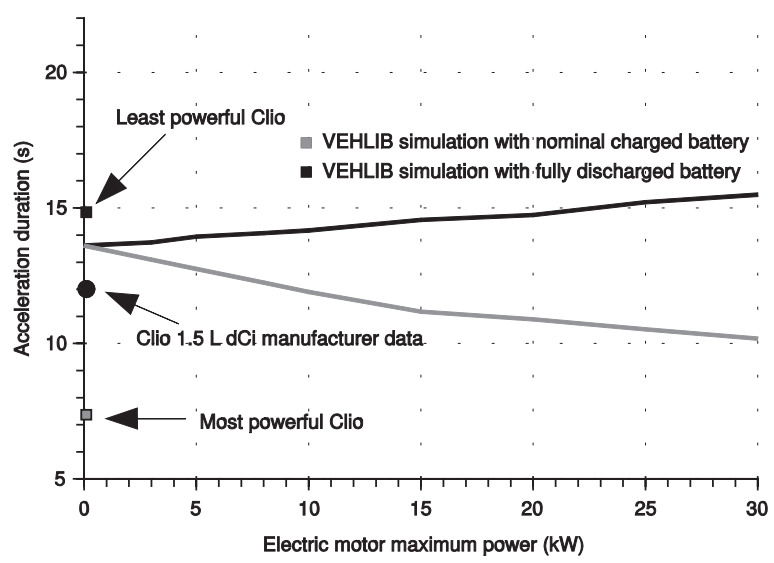

Fig. 5 0-100 km/h acceleration without ICE downsizing

\section{BACKWARD OPTIMIZATION TOOL KOALA}

The step after the sizing of a hybrid vehicle is the evaluation of its minimum fuel consumption. But this consumption is very dependant on the implemented energy management law. That is why we had the idea to get rid of this degree of freedom: the goal was to 
judge each vehicle with the best energy management law possible to conceive. That signify to find the optimal control for each vehicle. As we said earlier in $\S$ II, it is a great advantage to know the rolling conditions in advance.

So we have developed a specific optimization software, named KOALA. Its objective is the determination of optimal energy management laws as regards consumption, for a given $\mathrm{HEV}$ and vehicle usage.

It is important to notice that all the consumption results given by KOALA are maximum potential gains, because they cannot be reached in a real vehicle (using online strategies). The two main reasons are the following:

(1) Firstly, the optimal strategies given by KOALA are computed offline with the a priori knowledge of the entire driving schedule;

(2) Secondly, neither comfort constraints nor highly dynamic phenomena (e.g. clutch opening modelling) are taken into account. The reason for these simplifications is either mathematical complexity or combinatory explosion.

But, thanks to its reasonable computation speed and its flexibility, KOALA enables us to:

(1) Evaluate the minimum consumption attainable by an ideal online strategy, for any vehicle and for any sage. This enables a clear comparison between different hybrid vehicles, in term of fuel economy. Particularly it allows us to forecast the best sizing for a given architecture and set of components. Moreover, it allows us to evaluate the performance of any online strategy (by comparison with the optimum potential).

(2) From the optimal strategies given by KOALA, we can deduce online energy management laws. A systematic approach has been developed in LTE, using neural networks ( see $^{\circ} \S 6$ ).

The vehicle model and the equations of the optimization problem are detailed in a to-be-published paper in International Journal of Vehicle Design.

The solving of this optimization problem can be managed in two ways. The first method is variation calculation [Culioli, 1994], used by [Dubray, 2002, Delprat, 2002]; the second method, dynamic programming based upon Hamilton-Bellman-Jacobi (HBJ) functional equation, or more generally Bellman optimality principle [Borne et al., 1990, Culioli, 1994, Lin et al., 2004].

The advantages of the HBJ method, used for example by [Bouchard, 2002, Rimaux et al., 1999, Meaude Venot, 2001], are the following:

(1) Easy control of the total deviation of State of charge (SOC) on the driving schedule $(\triangle \mathrm{SOC}=$ final SOC - initial SOC);
(2) Possible simple introduction of instantaneous constraints (e.g. Start/Stop). The abilities of the power train are easily controlled at each time;

(3) Facilities are naturally given to keep discrete measurements made on engine testbeds. It is not compulsory to compute analytic approximation of efficiency maps;

(4) No limitation due to analytic formulation appears. For example we can easily introduce real-time phenomena such as clutch slip.

However the main drawback of the HBJ method is the impossibility of taking into account complex battery models using parameters depending on SOC, because of combinatory explosion [Lin et al., 2004].

Generally speaking, vehicle models used in global optimization process cannot take into account fast transient phenomena. It is not important since we have been focusing here on the energetic assessment on a driving schedule (the most important thing in modelling was to take into account inertia, that is the case). Usually, time sample for normalized and actual use driving schedules is $1 \mathrm{~s}$. This is also our choice in the scope of this article, as it leads to precise enough results.

The KOALA tool resolution algorithm is based upon HBJ equation. We have implemented the following possibilities:

(1) Gear shift optimization;

(2) Start/stop;

(3) Optimal auxiliaries power management;

(4) Serial flow (direct energy transfer from ICE to battery, this is the case today on classical vehicles) or not;

(5) Energy recovery (energy transfer from wheel to battery in deceleration phases) or not.

By allowing or forbidding some of these possibilities, it is possible to split the maximum potential gain in different parts to know the exact origin of the consumption decrease. On Figure 6 we have represented the variation of the potential consumption gains and their decomposition, depending on electric motor maximum power, as well as this precise decomposition. For this figure, consumption is evaluated on NEDC. Usually this cycle is performed with cold start. Since we can not take into account the engine temperature rise in the optimization scope, we assume a start in nominal temperature conditions. Each point abscissa on the graph corresponds to a particular sizing of the powertrain: a given EM maximum power, and the downsized ICE so as to meet the specification book. Each consumption point on this figure corresponds to a global optimization with known-inadvance driving schedule. The bottom of the light area corresponds to the minimal consumption that is possi- 


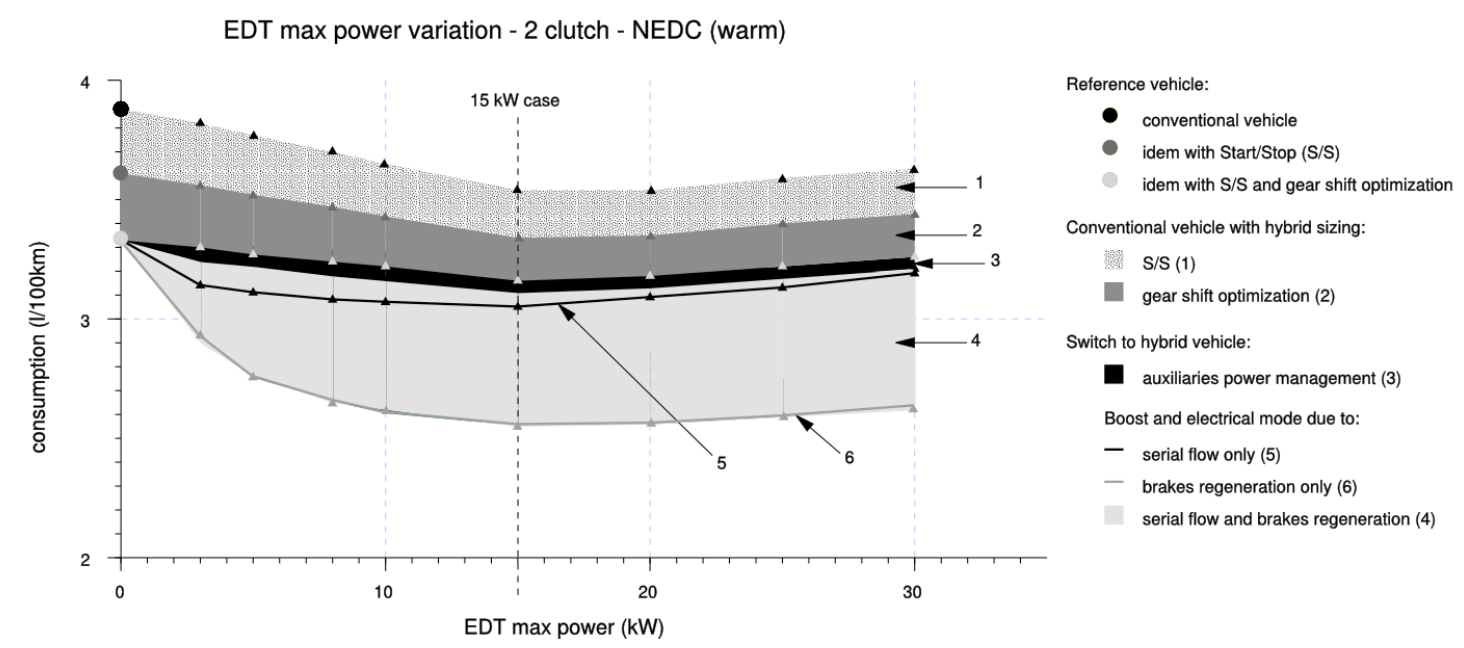

Fig. 6 Influence of the electric motor maximum power on the consumption gain for the case of ICE downsizing

ble to reach with the current vehicle and the best-ever control. On NEDC, the minimum consumption is attained for about $15 \mathrm{~kW}$ of electric motor power. It corresponds to an optimum compromise between brakes regeneration and ICE downsizing gain (adaptation of the efficiency map to the driving schedule). Above this power, the EM and battery weights increase, without subsequent brakes regeneration gain, and without further downsizing of the ICE, because of the minimal engine power constraint (to keep reasonable steadystate performances on highway). This prevents the consumption to keep on decreasing. Here is the split of different gains for an EM power of $15 \mathrm{~kW}$ :

(1) The top of the start/stop (S/S) area (top of area 1) corresponds to a hybrid vehicle used without its hybrid functions $(3.54 \mathrm{l} / 100 \mathrm{~km}$ at $15 \mathrm{~kW})$. If we allow to perform $\mathrm{S} / \mathrm{S}$, the consumption gain compared to $3.541 / 100 \mathrm{~km}$ is about $5 \%$ (bottom of area 1);

(2) Moreover, if we allow gear shift optimization (in the case of robotized gearbox), we obtain an additional gain of $5 \%$ (bottom of area 2 );

(3) Then, if we perform auxiliaries power management (see $\S 2$ ), we obtain a gain of about $2 \%$ (bottom of dark area 3). If the auxiliaries power had been higher, this gain would have been greater;

(4) The greatest gain is due to brakes regeneration, that is about $15 \%$ in our case (difference between the bottom of dark area 3 and line 6).

(5) It appears that the serial flow does not permit to obtain a significant additional gain. Indeed, examine the consumption difference between the bottom of dark area 3 and line 5: it is very slight. Likewise, examine the difference between line 6 and the bottom of light area 4 . The reason for the slight gain brought by serial flow is that it implies poor global efficiency for the vehicle (the energy flows through the ICE, electric motor, power electronics and battery). Nevertheless, sometimes serial flow has to be used, to prevent the battery from a deep discharge.

The reader will find a detailed explanation and analysis of this figure, as well as more parameter variations (EM maximum efficiency, and auxiliaries' power) in the literature [Scordia et al., 2004].

\section{VI NEURAL NETWORK APPROACH 6.1 A brief review concerning the elaboration of en- ergy management laws}

A tool such as KOALA described in $\S 5$ cannot be used in a real time process due to too much high computation times. So, even if it is possible to predict the future of the rolling conditions in one way or another, the command latency time is peremptory, at least for the current computational possibilities. Other methods must be found.

A first kind of approach is empirical conception of online energy management laws. A great number of such methods can be found in the literature [Vaccaro et al., 2004; Forgez et al., 2000; Wang et al., 2001; Van den Tillaart, 2000]. Among these, there are a lot use fuzzy logic and derived methods. Obviously all these laws have a trend towards the battery discharge increase when the state of charge rises, so as to keep state of charge in the range specified by the battery manufacturer. We may reproach the fact that these empirical laws are difficult to justify, and have to be precisely tuned to fit every particular case. Nevertheless they can entail good results. It is the case for thermostatic law used in [Delprat, 2002], for a full hybrid vehicle. A second kind of approach is management laws based on the analysis of the energy flow in the powertrain. Let us quote Loss Minimization Strategy (LMS) 
and Equivalent Consumption Minimization Strategy (ECMS). The principle of LMS [Dauphin Tanguy, 1999] is to choose the gearbox ratio and the power sharing that minimize the total losses in the powertrain. To be able to control battery state of charge, it is mandatory to introduce an extra constraint; otherwise the SOC would only decrease to minimize fuel consumption. ECMS is based on the consideration that, in charge sustaining mode, the only way to keep the battery SOC is to use serial flow, or brakes regeneration. So, a consumption equivalent to the future charge is computed permanently. The goal is to minimize the sum of the instantaneous consumption and the future consumption.

The third kind of energy management laws are based on the global optimization results. Their implementation can be more or less direct:

(1) Rather direct if the vehicle model used in the global optimization algorithm is sufficiently accurate (no instantaneous gearbox ratio shifting), and if the rolling conditions are known in advance. Then it is possible to keep in memory the optimum command all along the route, or use preestablished maps. This is the case for serial hybrid buses [Dubray, 2002];

(2) Rather indirect otherwise. It is possible to derive some algorithms from the original global optimization one [Delprat, 2002; Meaude Venot, 2001]. It is also possible to analyse the global optimization results to derive some expert online management laws.

In the scope of this paper, we will focus on a neural network approach. The goal is to learn KOALA global optimization results offline, and use this learning to control the vehicle online. So this approach is in the third category, and rather indirect.

\subsection{Radial Basis Function - Dynamic Decay Adjust- ment (RBF-DDA) network presentation}

One family of neural networks is progressive networks, for which the number of neurons is not set before the learning phase. In this family, let us consider classification networks, rather than approximation ones. In this case, each influence zone corresponds to a given category for the output, each represented by a neuron in the hidden layer of the network. One of these neural networks is RBF-DDA one, that moreover performs Bayesian classification, that is to say it takes into account the probability of each considered input vector during the learning phase [Michael et al., 1995]. In the frame of our study, we have used the open source software Stuttgart Neural Network Simulator, which we adapted to show generalization scope equal to infinite. We also implement a pruning proce- dure to suppress less significant hidden layer neurons.

\subsection{Choice of the NN inputs and output}

For the vehicle simulated in VEHLIB, the state variables are vehicle speed and battery SOC:

(1) The speed is regulated by the driver, simulated by a PID controller;

(2) The battery SOC, that has to be reproduced as close as possible to the SOC optimal curve computed in global optimization tool KOALA.

The analysis of the global optimization results allows us to define the parameters that have the greatest influence on the battery SOC behaviour. So they are the inputs of our neural network:

(1) The state of charge difference between the beginning and the end of the considered driving cycle during the learning phase (offline). During the use phase (online), we obviously cannot define what the end of the driving cycle is; so, to control the battery state of charge during online use, this input is taken as the opposite of the instantaneous state of charge;

(2) The instantaneous power at the wheels, that we have split into torque and speed parts. This allows us to obtain more information from the optimization results;

(3) The auxiliaries power. If it is very high, at the extreme it could prevent us from working in charge sustaining mode.

The output of the neural network has to allow the complete command of the hybrid powertrain. For the considered architecture, this output vector could be the gearbox ratio, the electric motor power, and the state of the ICE (on or off). Nevertheless, the gearbox ratio is computed instantaneously, and independently of the neural network, so as to maximize the efficiency of the ICE and EM. So the neural network will only furnish EM power to apply, and the state of the ICE. Note that the state of the clutches (opened or closed) can be easily deduced from the ICE state, independently of the neural network.

\subsection{Learning example: optimal SOC curve reproduc- tion for the NEDC cycle}

The Figure 7 shows in light tone the KOALA SOC optimal curve on the NEDC driving schedule (zero SOC variation on the cycle). Here only wheel speed and torque are considered for the learning and use of the NN. The response of the neural network in the use phase is represented on the same figure in dark tone. Compared to the learning curve, there are several differences:

(1) A large discharge is performed less before time equal to $100 \mathrm{~s}$, that does not appear in the learn- 

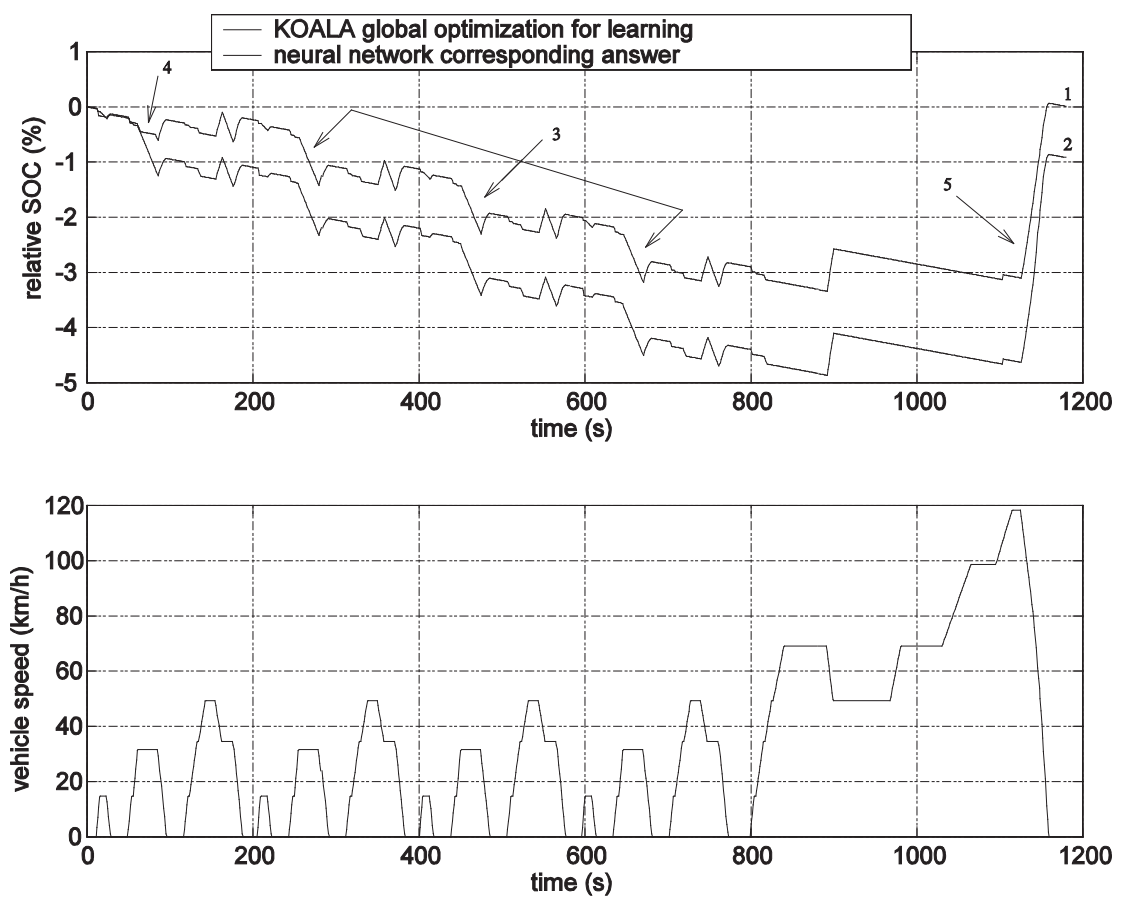

Fig. 7 NEDC optimal SOC curve learning and corresponding answer

ing. This is due to the fact that the NN performs a Bayesian classification during the learning phase, and so entails the most common class in use phase.

Indeed, the three arrows placed on the learning curve show three identical all-electric behaviour of the vehicle, whereas the first (vertical arrow) is realized with the ICE working. So the all-electric mode appears to be more probable during the learning phase; then it is chosen during the use phase;

(1) The SOC gap between the neural net and the learning curve increases until the end of the driving schedule. This is due to the sampling in twenty classes of the EM power;

(2) The brakes regeneration is larger in use phase than in learning phase. This is due to the SOC precision in KOALA during the global optimization. Indeed the learning curve underestimates slightly the regeneration potential. Nevertheless, this approximation has been carefully studied: the corresponding consumption error compared to the real optimum is quite small, around 1 to $2 \%$.

The rise in consumption between learning and use phases remains smaller than $3 \%$. This quantifies the consumption rise due to the fact that the driving cycle is no more known.

The learning has been extended to a larger learning base, and the use on unknown cycles (i.e. not in the learning base). Some results are presented in Table 2. The learning is performed on HYZEM driving schedules (urban, road, and highway, see ${ }^{\circ} \S 2$ ), and the use on HYZEM urban and road, ARTEMIS urban and road. The average rise due to not known in advance driving cycle is about $5 \%$. For these figures, we keep the KOALA model.

The resulting number of neurons is several thousand.

\section{HARDWARE-IN-THE-LOOP EXPERIMEN- TATION RESULTS}

INRETS owns a dynamic test bench that allows to reduce the efforts required to test a hybrid vehicle. Some components are simulated by a built-in software (clutch 2, gearbox, differential), while others are physically on the bench (clutch 1, ICE, EM). The road resistance and the aerodynamic drag are also simulated by the electric generator with a power of $120 \mathrm{~kW}$. This

Table 2 Consumption (L/100 km) increase due to unknown-in-advance driving cycle.

\begin{tabular}{lcccc}
\hline Use cycle & Urban HYZ. & Road HYZ. & Urban ART. & Road ART. \\
\hline Optimal consumption & 2.70 & 3.10 & 3.25 & 2.91 \\
\hline Online consumption & 2.87 & 3.29 & 3.45 & 3.03 \\
\hline Increase (\%) & 6.3 & 3.1 & 6.2 & 4.1 \\
\hline
\end{tabular}




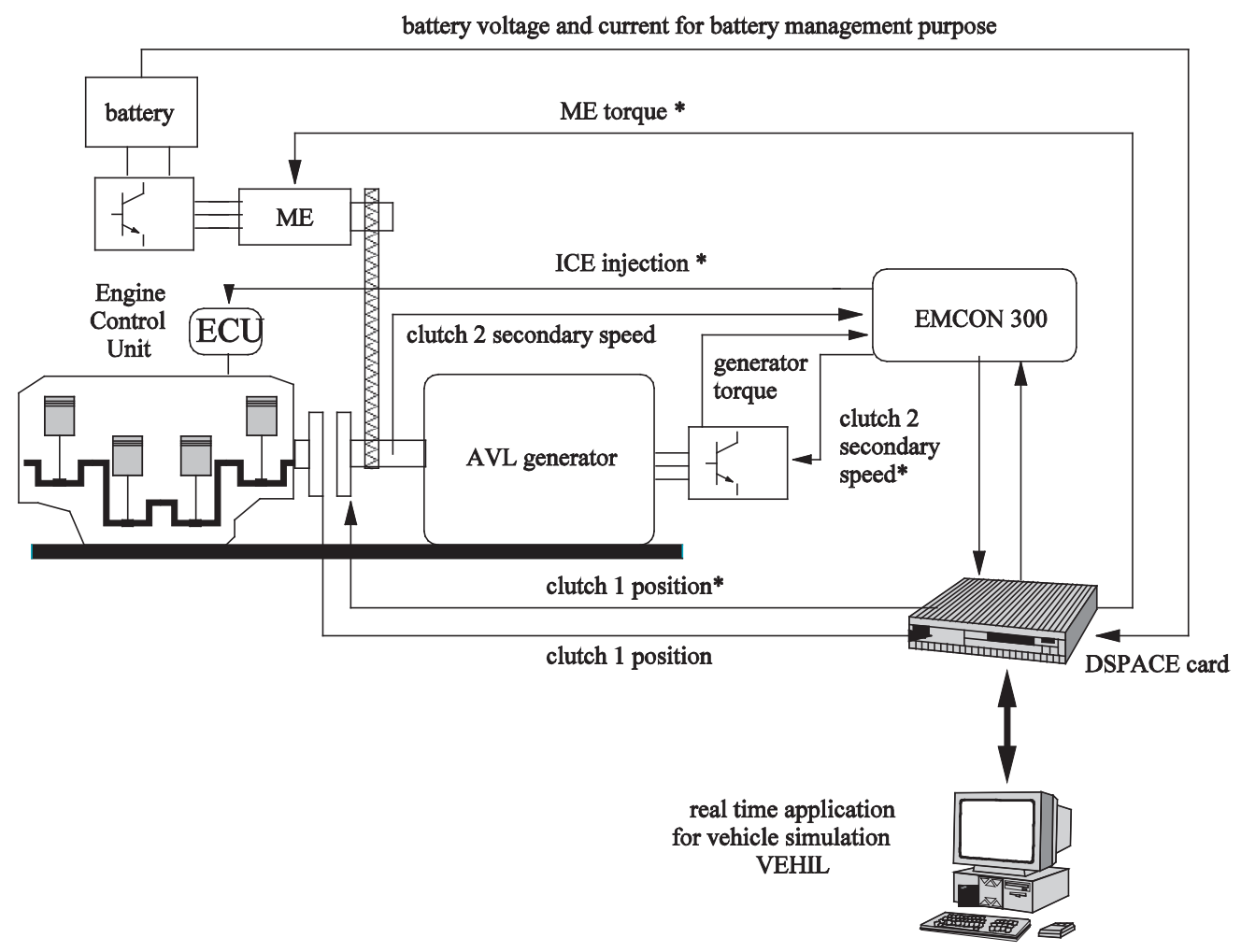

Fig. 8 INRETS' HIL test bench

approach enables us to [Jeanneret et al., 2004]:

(1) Muster our efforts to study the physic components;

(2) Quickly adapt the simulated components to our requirements;

(3) Obtain a good reproducibility of the experiments. The system is represented on Figure 8. An HIL version of VEHLIB named VEHIL is used to control the bench.

For the time being, the consumption measured on the test bench with a neural network control is $3.6 \mathrm{l} / 100$ $\mathrm{km}$ on the NEDC driving schedule, so an improvement of $14 \%$ compared to the reference thermal vehicle (the conventional Renault Clio $1.51 \mathrm{dCi}$ ). The deterioration of the consumption gain from step two to step four of our approach (see ${ }^{\circ} \S 3$ ) is summarized in Table 3.

Table 3 Consumption $(1 / 100 \mathrm{~km})$ and gain compared to the reference thermal vehicle on warm NEDC

\begin{tabular}{lccc}
\hline & Step 2 & Step 3 & Step 4 \\
\hline Reference vehicle & 3.02 & 3.01 & 4.09 \\
\hline Two clutch hybrid & 3.09 & 3.37 & 3.50 \\
\hline Improvement (\%) & 21.2 & 13.8 & 14.4 \\
\hline
\end{tabular}

\section{CONCLUSION AND OUTLOOKS}

We have described a global approach for the hybridi- zation of a reference thermal vehicle, from the optimal sizing to the online control, which resulting consumption must be as close as possible to the offline optimization results. The great advantage of our approach is its systematic aspect: by using global optimization in a derived way, we have shrunk the tuning procedure only to the minimum (essentially elaboration of comfort laws).

We currently study how it could be possible to reduce the number of neurons necessary to obtain a good performance improvement compared to the reference vehicle.

The obtained results on the test bench are satisfactory with regards to hybrid vehicle potential gain. This work is still in progress at INRETS.

\section{References}

Akihiro, K., A., Tetsua, and S. Shoichi, Drive force control of parallel series hybrid system, JSAE Review, Vol. 20, No. 3, 337-341, 1999.

André, M., Driving patterns analysis and driving cycles: European development of hybrid technology approaching efficient zero emission mobility (HYZEM), INRETS Report, LEN 9709, 1997.

André, M., The ARTEMIS European driving cycles for measuring car pollutant emissions, Science of the Total Environment, No. 334-335, 73-84, 2004.

Berthold, M., and J. Diamond, Boosting the performance of RBF networks with dynamic decay adjust- 
ment, Proceeding of the Advances in Neural Information Processing Systems, Vol. 7, 521-528, 1995.

Borne, P., G. Dauphin-Tanguy, J. P. Richard, F. Rotella, and I. Zambettakis, Control and optimisation of processes, Technip, 1990.

Bouchard, P., Efficiency optimisation of an automotive drive train, 9th Conferenceof Ph.D. School Systems, 2002.

Culioli, J. C., Introduction to the optimisation, Ellipse, 1994.

Delprat, S., Control strategies evaluation for parallel hybrid vehicle, Ph.D. Thesis of Valenciennes and Hainaut-Cambris University, 2002.

Dauphin-Tanguy, G., The Bond Graphs and their applications in mecatronic, Technique de l'ingénieur, 1999.

Dubray, A., Energy management laws adaptation for hybrid vehicles according to their mission profile, Ph.D. Thesis of National Institute Polytechnic of Grenoble, 2002.

European Union, Official Journal of the European Community, European Communities, 1990.

Forgez, C., G. Friedrich, and J. M. Biedinger, Method to find the hybridization rate for a parallel hybrid electrical vehicle, Proceedings of Electric Vehicle Symposium 17, 2000.

Garcelon J., Implementing optimization in Advisor using VisualDOC, Proceedings of ADVISOR Users Conference, 2000.

Lin, C. C., Z. Filipi, L. Louca, H. Peng, D. Assanis, and J. Stein, Modelling and control of mediumduty hybrid electric truck, International Journal of heavy Vehicle Systems, Vol. 11, No. 3-4, 349-369, 2004.

Meaude-Venot, N., Modelling and control of a power train with low consumption. Example of Hybrid systems control problems, Ph.D. Thesis of Univeisity of Paris IX Dauphine, 2001.

Paganelli, G., Design and control of a drivetrain for parallel hybrid electric vehicles, Ph.D. thesis of Valenciennes and Hainaut-Cambris University, 1999.

Rimaux, S., M. Delhom, and E. Combes, Hybrid vehicle powertrain: Modelling and control, Proceeding of Electric Vehicle Symposium 16, 1999.

Scordia, J., M. Desbois-Renaudin, and R. Trigui, Hybrid power train sizing and potential consumption gains, IEEE Proceeding of Vehicular Power and Propulsion, 2004.

Scordia, J., M. Desbois-Renaudin, R. Trigui, B. Jeanneret, and F. Badin, Global optimization of energy management laws in hybrid vehicles using dynamic programming, International Journal of Vehicle Design, Vol. 39, No. 4, 349-367, 2005.

TNO Automotive, MATADOR: Management tool for the assessment of driveline technologies and research, 2000.

Trigui, R., B. Jeanneret, and F. Badin, System modelling of hybrid vehicles in order to predict their energy and dynamic performance: Building VEHLIB library, Recherche Transports Sécurité, Vol. 83, 129-150, 2004.

Trigui, R., B. Jeanneret, B. Malaquin, F. Badin, C. Plasse, Hardware in the loop simulation of a diesel parallel mild-hybrid electric vehicle, 2007 IEEE Vehicular Power and Propultion, 2007.

Vaccaro, A., and D. Villacci, Prototyping of a fuzzy based energy manager for parallel hybrid electric vehicle, Proceeding of European Ele-Drive Transportation Conference \& Exhibition, 2004.

Wang, A., Y. Chen, and R. Zhang, A novel design of energy management system for hybrid electric vehicles using evolutionary computation, Proceeding of Electric Vehicle Symposium 18, 2001.

(Received September 24, 2008; accepted April 30, 2009) 